\title{
Alternativas de controle químico do capim-amargoso resistente ao glyphosate, com herbicidas registrados para as culturas de milho e algodão
}

\author{
Chemical control alternatives for glyphosate-resistant sourgrass using \\ hebicides indicated for corn and cotton crop
}

Marcel Sereguin Cabral de Melo ${ }^{1 *}$, Leonardo José Frinhani Noia da Rocha ${ }^{2}$, Caio Augusto de Castro Grossi Brunharo ${ }^{3}$, Danilo Carvalho Pereira da Silva ${ }^{4}$, Marcelo Nicolai $^{5}$, Pedro Jacob Christoffoleti ${ }^{4}$

Resumo - Nos sistemas de produção agrícolas no Brasil é comum a rotação de culturas soja, milho e/ou algodão, todas resistentes ao herbicida glyphosate; o que favorece a seleção de populações de capim-amargoso (Digitaria insularis) resistente ao glyphosate. Para as culturas de milho e algodão, pouco se sabe sobre alternativas para manejo químico para $D$. insularis. Dessa forma, torna-se pertinente a investigação da eficácia de herbicidas de mecanismos de ação alternativos ao glyphosate, aplicados em condições de pré e pós-emergência, para o controle do biótipo resistente de capim-amargoso ( $D$. insularis), recomendados para as culturas do milho e algodão. O experimento foi desenvolvido em casa-de-vegetação, durante os meses de setembro a dezembro de 2014, utilizando um biótipo de D. insularis resistente ao glyphosate. O delineamento estatístico utilizado foi de blocos ao acaso com quatro repetições. A aplicação aconteceu quando as plantas de capim-amargoso estavam no estádio de 1 a 2 perfilhos e em pré-emergência com diferentes herbicidas. Os tratamentos que obtiveram melhores resultados em condições de pós-emergência da planta daninha foram os herbicidas nicosulfuron, imazapic + imazapyr, atrazine, haloxifop-methyl e tepraloxydim. Os herbicidas atrazine, isoxaflutole, S-metolachlor, clomazone, diuron e flumioxazin se apresentaram como pré-emergentes eficazes para o controle desta espécie.

Palavras-chave: resistência, associações, graminicidas, pré-emergência, pós-emergência

Abstract - In Brazil cropping systems is common a rotation between soybean, corn and / or cotton, all resistant to glyphosate, which favor the selection of glyphosate resistant sourgrass populations (Digitaria insularis). Little is known about alternative chemical control of $D$. insularis registered for the corn and cotton crops. Thus, it is relevant to investigate herbicides with alternative mode of action, applied in pre and post-emergence conditions, to be used for efficient control of the glyphosate resistant sourgrass biotype (D. insularis). The experiment was conducted in greenhouse, from

Recebido: Julho 04, 2017. Aceito: Outubro 09, 2017.

${ }^{1}$ Bayer, Avenida Dr. Roberto Moreira, 5005, Setor EAE, Recanto dos Pássaros, CEP 13148-914, Paulínia, SP, Brasil.

E-mail: melomsc@yahoo.com.br

${ }^{2}$ Universidade Federal do Espírito Santo - UFES, Alegre, ES, Brasil. E-mail: leofrinhanirocha@gmail.com

${ }^{3}$ University of California, Davis, CA, Estados Unidos da América. E-mail: cabrunharo@ucdavis.edu

${ }^{4}$ Escola Superior de Agricultura Luiz de Queiroz - ESAlQ, Universidade de São Paulo - USP, Piracicaba, SP, Brasil. E-mail: danilo_carvalho79@hotmail.com; pedrochristoffoleti@gmail.com

${ }_{5}^{5}$ Agrocon Assessoria Agronômica, Santa Bárbara D’Oeste, SP, Brasil. E-mail: mnicolai2009@gmail.com 
September to December of 2014, using a glyphosate resistant D. Insularis biotype. The statistical design was randomized blocks with four replications. The application occurred when sourgrass plants were at the 1 to 2 tillers stage for post-emergent and at pre-emergence for the pre-emergent herbicides. The treatments which showed the best results in post-emergence conditions were the nicosulfuron, imazapic + imazapyr, atrazine, haloxyfop-methyl and tepraloxydim herbicides. Atrazine, isoxaflutole, S-metolachlor, clomazone, diuron and flumioxazin presented themselves as effective pre-emergents for controlling this species.

Keywords: resistance, associations, graminicides, pre-emergence, post-emergence

\section{Introdução}

$\mathrm{Na}$ agricultura moderna as moléculas de herbicidas vêm substituindo a força humana, animal e mecânica no controle de plantas daninhas em lavouras de forma eficaz, evitando perdas de rendimento das culturas, e assim contribuindo para maior produção global de alimentos (Powles e Yu, 2010).

O glyphosate é um herbicida de grande importância mundial, sendo utilizado por muitos anos no controle de plantas daninhas anuais e/ou perenes em diversos sistemas de produção. Com o advento e a ampla adoção de culturas geneticamente modificadas, ocorreu uma nova revolução na agricultura (Powles e Yu, 2010). Este fenômeno resultou na dependência, em alguns países, do glyphosate como principal forma de manejo das plantas daninhas. Esse herbicida possui amplo espectro de controle, ajustável a diferentes estádios de desenvolvimento das espécies daninhas alvo, baixa volatilidade (Webster e Sonoskie, 2010) e está classificado entre os herbicidas menos tóxicos para animais (Duke e Powles, 2008).

A dependência do glyphosate desacelerou a pesquisa e desenvolvimento de novas moléculas de herbicidas, porém a alta pressão de seleção imposta pelo uso do glyphosate gerou um processo de seleção de diversos biótipos de espécies de plantas daninhas resistentes no mundo (Heap, 2017).

Uma das plantas daninhas que são resistentes ao glyphosate é o capim-amargoso (Digitaria insularis).
O primeiro caso relatado sobre o surgimento de um biótipo de $D$. insularis resistente ao glyphosate, veio do Paraguai, no ano de 2006 (Heap, 2017). $\mathrm{O}$ fato de ser uma planta que pereniza nas áreas agrícolas, produz alta quantidade de sementes e tem um vigoroso desenvolvimento vegetativo, associado ao fato que o produtor ter aumentado a dose de glyphosate em função da aplicação em estádios tardios de desenvolvimento desta planta daninha, eram indicativos do risco desta planta daninha desenvolver resistência ao glyphosate (Duke e Powles, 2008; Powles e Yu, 2010).

A reintrodução e manutenção da diversidade de estratégias de manejo, como herbicidas alternativos ao glyphosate para o controle de plantas daninhas são práticas necessárias para impedir ou retardar o surgimento de biótipos resistentes e preservar o glyphosate como ferramenta de manejo (Powles, 2008).

Em estudo realizado nos Estados Unidos com mais de 1.000 agricultores, que cultivam milho, algodão e soja, revelou-se que o uso de múltiplos herbicidas com diferentes mecanismos de ação era a prática menos adotada no manejo da resistência (Frisvold et al., 2009). Os relatos mais comuns dos agricultores dos motivos pelos quais eles não adotavam essa técnica eram os seguintes: (i) alguns produtores acreditam que o uso de mais de um herbicida com diferentes mecanismos de ação aumentava o custo de produção e o controle de plantas daninhas resistentes era incerto (Hurtley et al., 2009); (ii) alguns agricultores acreditam que controlar 
plantas daninhas resistentes está além de suas capacidades (Wilson et al., 2008); (iii) outros agricultores acreditam que a indústria criará novos herbicidas capazes de controlar biótipos resistentes (Llewellyn, 2007). Em todos os casos os agricultores estão equivocados.

O uso de herbicidas alternativos em associação com o glyphosate visando o aumento do número de espécies de plantas daninhas controladas em uma mesma aplicação tem sido tradicionalmente realizado nas culturas da soja e algodão, com boa eficiência no controle de plantas daninhas resistentes ao glifosato, como a buva (Conyza bonariensis) (Yamauti et al., 2010; Gemelli et al., 2013). Todavia, são poucos herbicidas registrados para o controle de D. insularis no Brasil, sendo estes recomendados para aplicação em pré-emergência ou em estádios precoces de desenvolvimento desta espécie (Gemelli et al., 2012).

Nesse contexto, este trabalho teve como objetivo avaliar herbicidas alternativos ao glyphosate, eficazes para o controle do biótipo resistente de capim-amargoso (D. insularis), para serem utilizados nas culturas de milho e algodão, tanto em condições de pré-emergência da planta daninha, como em condições de pós-emergência inicial.

\section{Material e métodos}

O experimento foi desenvolvido em casa-devegetação, com irrigação diária de aproximadamente $4 \mathrm{~mm}$ distribuídos durante o dia, no período de setembro a dezembro de 2014, utilizando a população resistente ao glyphosate (Melo, 2015) oriunda do município de Campo Florido em Minas Gerais, cujas coordenadas geográficas são, Sul 1945'48.80"' Oeste 48³4'19.47'.

Para o experimento com herbicidas pós-emergentes as unidades experimentais foram compostas por vasos plásticos de $1 \mathrm{~L}$, preenchidos com substrato adubado onde foi transplantada uma planta de capim-amargoso por vaso. As plantas se desenvolveram até o estádio de 1 a 2 perfilhos quando foram aplicados os tratamentos herbicidas descritos na Tabela 1.

O delineamento experimental utilizado foi de blocos ao acaso com quatro repetições, sendo 11 tratamentos herbicidas e uma testemunha sem aplicação.

No experimento com herbicidas pré-emergentes as unidades experimentais foram compostas por vasos plásticos de $1 \mathrm{~L}$, preenchidos com solo de textura argilosa adubado, cujas características químicas

Tabela 1. Lista de tratamentos herbicidas e doses, aplicados em condições de pós-emergência do capim-amargoso (Digitaria insularis) com seus respectivos mecanismos de ação.

\begin{tabular}{|c|c|c|c|}
\hline Tratamentos & Dose (g ha ${ }^{-1}$ i.a. $)^{*}$ & $\begin{array}{c}\text { Adjuvante** } \\
\text { (Concentração v/v) }\end{array}$ & Nome comercial \\
\hline 1 - Testemunha sem aplicação & - & - & - \\
\hline $2-$ Nicosulfuron $^{\mathrm{M}}$ & 60 & Assist a $0,5 \%$ & Sanson $40 \mathrm{SC}$ \\
\hline $3-$ Mesotrione $^{\mathrm{M}}$ & 192 & Assist a $0,5 \%$ & Callisto $480 \mathrm{SC}$ \\
\hline $4-$ Tembotrione $^{\mathrm{M}}$ & 100,8 & Assist a $0,5 \%$ & Soberan $420 \mathrm{SC}$ \\
\hline 5 - Imazapic + Imazapyr ${ }^{1 \mathrm{M}}$ & $52,5+17,5$ & Assist a $0,5 \%$ & Onduty $525+175 \mathrm{WG}$ \\
\hline 6 - Carfentrazone ${ }^{\mathrm{MA}}$ & 12 & Assist a $0,5 \%$ & Aurora $400 \mathrm{CE}$ \\
\hline 7 - Atrazine $^{\mathrm{M}}$ & 3000 & Assist a $0,5 \%$ & Gesaprim $500 \mathrm{SC}$ \\
\hline $8-$ Flumioxazin $^{\mathrm{A}}$ & 30 & Assist a $0,5 \%$ & Flumyzin $500 \mathrm{WG}$ \\
\hline 9 - Haloxifop-methyl ${ }^{\mathrm{A}}$ & 60 & Assist a $0,5 \%$ & Verdict R $120 \mathrm{CE}$ \\
\hline 10 - Trifloxisulfuron-sodium ${ }^{\mathrm{A}}$ & 7,5 & Assist a $0,5 \%$ & Envoke $750 \mathrm{WG}$ \\
\hline $11-\mathrm{MSMA}^{\mathrm{A}}$ & 790 & Assist a $0,5 \%$ & Volcane $790 \mathrm{CS}$ \\
\hline 12 - Tepraloxydim ${ }^{\mathrm{A}}$ & 100 & Assist a $0,5 \%$ & Aramo $200 \mathrm{CE}$ \\
\hline
\end{tabular}


estão descritas na Tabela 2 e as físicas na Tabela 3. Foram semeadas 10 sementes de capim-amargoso por vaso. O delineamento estatístico utilizado foi de blocos ao acaso com quatro repetições, sendo 7 tratamentos herbicidas e uma testemunha sem aplicação especificados na Tabela 4.

Os tratamentos herbicidas foram aplicados através de pulverizador costal pressurizado por $\mathrm{CO}_{2}$, trabalhando a pressão de 30 psi, regulado para uma vazão de $200 \mathrm{~L} \mathrm{ha}^{-1}$. A barra de aplicação possuía 2 bicos de aplicação equipados com pontas de pulverização do tipo "leque" XR110:02 VS.

Após a aplicação dos tratamentos herbicidas foram realizadas avaliações percentuais visuais de controle aos 7, 14, 21 e 28 dias após a aplicação (DAA) no experimento com herbicidas pós-emergentes e aos 20, 60 e 80 dias após a aplicação (DAA) para o experimento com herbicidas pré-emergentes. As avaliações visuais percentuais têm como referência sendo $0 \%$ a ausência total de sintomas e $100 \%$ morte da planta, conforme metodologia proposta pela Sociedade Brasileira da Ciência das Plantas Daninhas (SBCPD, 1995). Após as últimas avaliações visuais de cada experimento foram coletadas a parte aérea das plantas por vaso, para secagem em estufa de circulação de ar forçada a $60{ }^{\circ} \mathrm{C}$, durante dois dias, seguido da pesagem da massa seca.

Para a análise estatística, os dados apresentados foram submetidos à análise de variância pelo teste F e as médias comparadas pelo teste de Tukey a $5 \%$ de significância.

Tabela 2. Características químicas do solo utilizado no experimento de alternativas de controle com herbicidas pré-emergentes. Piracicaba, 2014.

\begin{tabular}{|c|c|c|c|c|c|c|c|c|c|}
\hline Profundidade & pH & M.O. & $\mathbf{P}$ & $\mathbf{K}$ & $\mathbf{C a}$ & Mg & SB & CTC & $\mathbf{V}$ \\
\hline (cm) & $\mathrm{CaCl} 2$ & g dm-3 & $\overline{\mathrm{mg} \mathrm{dm}-3}$ & \multicolumn{6}{|c|}{ mmolc dm-3 } \\
\hline $0-20$ & 6,4 & 14 & 17 & 3 & 48 & 18 & 69,1 & 87,2 & 79 \\
\hline
\end{tabular}

Fonte: Departamento de Ciências do Solo, ESALQ/USP.

Tabela 3. Propriedades físicas do solo utilizado no experimento de alternativas de controle com herbicidas pré-emergentes (em g. $\left.\mathrm{kg}^{-1}\right)$.

\begin{tabular}{|c|c|c|c|c|c|}
\hline $\begin{array}{c}\text { Profundidade } \\
\text { (cm) }\end{array}$ & Areia & Silte & Argila & $\begin{array}{c}\text { Classe } \\
\text { textural } \\
\end{array}$ & Origem \\
\hline $0-20$ & 305 & 123 & 572 & Argiloso & Piracicaba \\
\hline
\end{tabular}

Fonte: Departamento de Ciências do Solo, ESALQ/USP.

Tabela 4. Lista de tratamentos herbicidas e doses, aplicados em condições de pré-emergência do capim-amargoso (Digitaria insularis) com seus respectivos mecanismos de ação.

\begin{tabular}{|c|c|c|}
\hline Tratamentos & Dose ( g ha $^{-1}$ i.a.)* & Nome comercial \\
\hline 1 - Testemunha sem aplicação & - & - \\
\hline 2 - Amicarbazone ${ }^{\mathrm{M}}$ & 280 & Dinamic $700 \mathrm{WG}$ \\
\hline 3 - Atrazine $^{\mathrm{M}}$ & 3.000 & Gesaprim $500 \mathrm{SC}$ \\
\hline 4 - Isoxaflutole ${ }^{\mathrm{MA}}$ & 100,8 & Provence $750 \mathrm{WG}$ \\
\hline 5 - S-metolachlor ${ }^{\mathrm{M}}$ & $52,5+17,5$ & Dual Gold $960 \mathrm{CE}$ \\
\hline $6-$ Clomazone $^{\mathrm{A}}$ & 12 & Gamit $500 \mathrm{CE}$ \\
\hline $7-$ Diuron $^{\mathrm{A}}$ & 3.000 & Karmex $800 \mathrm{WG}$ \\
\hline $8-$ Flumioxazin $^{\mathrm{A}}$ & 50 & Flumyzin $500 \mathrm{WG}$ \\
\hline
\end{tabular}




\section{Resultados e discussão}

$\mathrm{Na}$ Tabela 5 estão representadas as médias de controle da planta daninha capim-amargoso (Digitaria insularis) resistente ao glyphosate, obtidos pela observação dos sintomas oriundos da aplicação dos tratamentos herbicidas pós-emergentes, descritos na Tabela 1. De acordo com o Ministério da Agricultura, Pecuária e Abastecimento, a média de controle que um herbicida deve promover para ser considerado viável e pleitear registro junto ao órgão citado é de no mínimo 80\% (MAPA, 2017). Conforme preconizado por Frans et al. (1986), o controle médio de um herbicida de $80 \%$ sobre uma determinada planta daninha é o mínimo necessário para que esta não mais interfira sobre a cultura de interesse. Dessa forma os tratamentos que obtiveram nota percentual de controle abaixo de $80 \%$, foram considerados como ineficazes.
No experimento com herbicidas pós-emergentes, os tratamentos que obtiveram melhores controles, não diferindo estatisticamente e apresentando médias de controle acima de $80 \%$ aos 35 DAA, foram nicosulfuron (60 $\mathrm{g} \mathrm{ha}^{-1}$ i.a.), imazapic + imazapyr (52,5 + 17,5 $\mathrm{g} \mathrm{ha}^{-1}$ i.a.), atrazine (3000 $\mathrm{g} \mathrm{ha}^{-1}$ i.a.), haloxyfop-methyl (60 $\mathrm{g} \mathrm{ha}^{-1}$ i.a.) e tepraloxydim (100 $\mathrm{g} \mathrm{ha}^{-1}$ i.a.) Para a avaliação da massa seca aos 35 dias após a aplicação dos tratamentos, os resultados foram semelhantes aos da avaliação de controle visual (Tabela 5), o que fez com que os tratamentos descritos acima se apresentem como boas alternativas de controle químico para o capim-amargoso.

Adegas et al. (2010), demonstraram que empregando clethodim, fluazifop-p-buthyl, fenoxaprop-pethyl, tepraloxydim, clethodim+fenoxaprop-pethyl, paraquat, haloxyfop-methyl e imazapyr em plantas de capim-amargoso com até dois perfilhos

Tabela 5. Controle percentual de capim-amargoso (Digitaria insularis) avaliado aos 7, 14, 21, 28 e 45 dias após a aplicação dos tratamentos herbicidas pós-emergentes (DAA), de forma visual e massa seca.

\begin{tabular}{|c|c|c|c|c|c|c|c|}
\hline \multirow{3}{*}{ Tratamentos } & \multirow{3}{*}{$\begin{array}{c}\text { Dose } \\
\text { g ha }^{-1} \text { i.a. }\end{array}$} & \multicolumn{6}{|c|}{ Capim-amargoso (Digitaria insularis) } \\
\hline & & \multicolumn{6}{|c|}{ Avaliações de controle (\%) } \\
\hline & & $7 \mathbf{D A A}^{* *}$ & 14 DAA $* *^{*}$ & $21 \mathbf{D A A}^{* *}$ & 28 DAA $^{* *}$ & 35 DAA** & $\mathbf{M S}^{2}$ \\
\hline 1 - Testemunha & - & $0 \mathrm{c}$ & $0 \mathrm{~d}$ & $0 \mathrm{e}$ & $0 \mathrm{~d}$ & $0 \mathrm{~d}$ & $20,85 \mathrm{f}$ \\
\hline $2-$ Nicosulfuron $^{* \mathrm{M}}$ & 60 & $50 \mathrm{ab}$ & $77,5 \mathrm{ab}$ & $88,7 \mathrm{a}$ & 98,7 & $97,5 \mathrm{a}$ & $0,34 \mathrm{a}$ \\
\hline $3-$ Mesotrione $^{* \mathrm{M}}$ & 192 & $37,5 \mathrm{~b}$ & $45 \mathrm{c}$ & $50 \mathrm{~cd}$ & $32,5 \mathrm{c}$ & $20 \mathrm{~cd}$ & 8,19 e \\
\hline $4-$ Tembotrione $^{* \mathrm{M}}$ & 100,8 & $30 \mathrm{bc}$ & $50 \mathrm{bc}$ & $45 \mathrm{~cd}$ & $35 \mathrm{c}$ & $31,2 \mathrm{bc}$ & 5,42 bcde \\
\hline 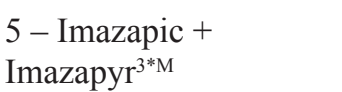 & $52,5+17,5$ & $61,2 \mathrm{ab}$ & $86,2 \mathrm{a}$ & 93,7 a & 98,7 & $100 \mathrm{a}$ & $0,14 \mathrm{a}$ \\
\hline 6 - Carfentrazone $\mathrm{e}^{* \mathrm{MA}}$ & 12 & $46,2 \mathrm{ab}$ & $48,7 \mathrm{c}$ & $30 \mathrm{~d}$ & $12,5 \mathrm{~d}$ & $15 \mathrm{~cd}$ & $7,98 \mathrm{e}$ \\
\hline $7-$ Atrazine $^{* \mathrm{M}}$ & 3000 & $43,7 \mathrm{~b}$ & $88,7 \mathrm{a}$ & $96,2 \mathrm{a}$ & $97,5 \mathrm{a}$ & $100 \mathrm{a}$ & $0,72 \mathrm{abc}$ \\
\hline 8 - Flumioxazin ${ }^{* A}$ & 30 & $50 \mathrm{ab}$ & $65 \mathrm{abc}$ & $63,7 \mathrm{bc}$ & $65 \mathrm{~b}$ & $55 \mathrm{~b}$ & 2,47 abcd \\
\hline 9 - Haloxifop-methyl ${ }^{* A}$ & 60 & $53,7 \mathrm{ab}$ & $70 \mathrm{abc}$ & $96,2 \mathrm{a}$ & $100 \mathrm{a}$ & $100 \mathrm{a}$ & $0,45 \mathrm{a}$ \\
\hline $\begin{array}{l}10 \text { - Trifloxisulfuron- } \\
\text { sodium*A }\end{array}$ & 7,5 & $48,7 \mathrm{ab}$ & $47,5 \mathrm{c}$ & $53,75 \mathrm{c}$ & $42,5 \mathrm{c}$ & $32,5 \mathrm{bc}$ & $6,89 \mathrm{de}$ \\
\hline $11-\mathrm{MSMA}^{* \mathrm{~A}}$ & 790 & $78,7 \mathrm{a}$ & $62,5 \mathrm{abc}$ & $52,5 \mathrm{c}$ & $37,5 \mathrm{c}$ & $27,5 \mathrm{c}$ & 5,54 cde \\
\hline 12 - Tepraloxydim *A & 100 & $45 \mathrm{~b}$ & $50 \mathrm{bc}$ & $82,5 \mathrm{ab}$ & $92,5 \mathrm{a}$ & $97,5 \mathrm{a}$ & $0,55 \mathrm{ab}$ \\
\hline DMS $^{4}$ & - & 33,01 & 28,26 & 20,77 & 16,63 & 26,55 & 4,94 \\
\hline CV $(\%)^{5}$ & - & 29,27 & 19,76 & 13,34 & 11,28 & 18,98 & 40,1 \\
\hline
\end{tabular}

${ }^{1}$ gramas de ingrediente ativo por hectare; ${ }^{2}$ Massa seca em gramas aos 35 DAA; ${ }^{3}$ mistura formulada de produtos; ${ }^{4}$ Diferença mínima significativa; ${ }^{5}$ Coeficiente de variação; ${ }^{M}$ Herbicida recomendado para a cultura do milho; ${ }^{\mathrm{A}}$ Herbicida recomendado para a cultura do algodão; ${ }^{\mathrm{MA}}$ Herbicida recomendado para as culturas do milho e algodão; *Assist $0,5 \%$; **Dias após a aplicação. 
é possível obter níveis de controle superiores a $90 \%$. O mesmo foi observado na avaliação dos herbicidas nicosulfuron, tepraloxydim, atrazine, haloxyfop-methyl e imazapic+imazapyr aos 35 dias após a aplicação.

Os tratamentos pós emergentes que não apresentaram um controle satisfatório aos 35 dias após a aplicação indicam que os mesmos não são recomendados para a espécie citada no estádio de desenvolvimento utilizado (Andrei, 2009; MAPA, 2017), sendo eles o mesotrione (192 $\mathrm{g} \mathrm{ha}^{-1}$ i.a.), tembotrione (100,8 $\mathrm{g} \mathrm{ha}^{-1}$ i.a.), carfentrazone (12 $\mathrm{g} \mathrm{ha}^{-1}$ i.a.), flumioxazin (30 $\mathrm{g} \mathrm{ha}^{-1}$ i.a.), trifloxisulfuron-sodium (7,5 $\mathrm{g} \mathrm{ha}^{-1}$ i.a. $)$ e MSMA (790 $\mathrm{g} \mathrm{ha}^{-1}$ i.a.).

A falha no controle devido ao estádio avançado de desenvolvimento da $D$. insularis, foi verificada também com o uso de mesotrione aplicado em pós-emergência da cultura do milho com intuito de controlar a rebrota de plantas de capim-amargoso. Este herbicida proporcionou controle médio de $70 \%$ aos 30 dias após a aplicação, porém não impediu o desenvolvimento do capim-amargoso até o final do ciclo da cultura do milho (Timossi, 2009).

Através da análise dos trabalhos desenvolvidos para manejar os biótipos de $D$. insularis resistentes ao glyphosate fica evidente que a utilização pontual de herbicidas inibidores da ACCase em pós-emergência poderá não ser a solução para o problema em todos os estádios de desenvolvimento desta espécie (Parreira et al., 2010). Também tem que ser considerado que a dependência do uso de um único mecanismo de ação para o controle do capim-amargoso, especialmente em áreas de resistência ao glyphosate, pode se constituir numa fonte de pressão de seleção para resistência aos herbicidas inibidores da ACCase (Gemelli et al., 2012).

Os graminicidas utilizados neste trabalho são os herbicidas pertencentes ao mecanismo de ação de inibição da acetil coenzima A (ACCase). Este mecanismo de ação congrega dois grupos químicos, os ariloxifenoxipropionatos, representados pelos herbicidas haloxyfop-methyl, fluazifop-p-butyl e fenoxaprop-p-ethyl e os ciclohexanodionas, com os herbicidas sethoxydim, tepraloxydim e clethodim. Independentemente do controle de D. insularis, os graminicidas pertencentes ao mecanismo de ação de inibição da ACCase não tem ação sobre espécies dicotiledôneas (Andrei, 2009), o que torna o uso de um graminicida isolado ideal apenas em áreas cuja infestação é composta exclusivamente por gramíneas, situação esta difícil de ocorrer em áreas agrícolas comerciais extensas e que ocupam diferentes localidades. A estratégia que se visualiza é a associação de graminicidas com o glyphosate.

Portanto, os herbicidas que apresentaram bom controle neste trabalho podem ter seu efeito potencializado quando usados em aplicações seqüênciais ou em complementação a ação promovida pelo glyphosate. Ferreira et al. (2010) avaliaram aplicações seqüenciais do herbicida amônio-glufosinato para o controle da planta daninha buva (Conyza spp.) resistente ao glyphosate e observaram que o amônio-glufosinato complementou a ação de outros produtos como metribuzin, glyphosate e paraquat. Moreira et al. (2007) também aponta o amônio-glufosinato como alternativa de manejo em associação ao glyphosate.

Com relação à associação entre graminicidas e o glyphosate, Vargas et al. (2005) utilizaram glyphosate e diferentes herbicidas inibidores da ACCase, de forma isolada e em aplicações seqüênciais, observando que há efeito da ação do glyphosate sobre a capacidade de controle dos graminicidas, tanto sobre biótipos suscetíveis, quanto sobre biótipos resistentes de L. multiflorum. Para Vargas et al. (2006) o clethodim é eficaz para o manejo de biótipos de L. multiflorum resistentes ao herbicida glyphosate, em estádios de desenvolvimento até o perfilhamento. Lingenfelter e Curran (2007) controlaram a planta daninha Muhlenbergia frondosa com o uso de glyphosate e dos graminicidas quizalofop, fluazifop, sethoxydim e clethodim. Barroso et al. (2014) controlou biótipos de $D$. insularis resistentes ao glyphosate utilizando herbicidas inibidores da ACCase isolados e em mistura com glyphosate, contudo não observou 
efeito sinérgico para os tratamentos em mistura quando comparados aos graminicidas isolados.

De acordo com Melo et al. (2012), os tratamentos herbicidas inibidores da ACCase em mistura com glyphosate são alternativas viáveis de controle para o biótipo resistente ao glyphosate de $D$. insularis, porém os herbicidas paraquat + diuron $\left(400+200 \mathrm{~g} \mathrm{ha}^{-1}\right.$ i.a.) e amônio-glufosinato (600 $\mathrm{g} \mathrm{ha}^{-1}$ i.a.) utilizados de forma isolada não são viáveis dependendo do estádio da $D$. insularis no momento da aplicação, sendo necessário ser associado ao glyphosate de forma sequencial para obterem bom controle.

Para o controle de $D$. insularis na cultura da soja, com o uso de herbicidas pós-emergentes alternativos ao glyphosate, os herbicidas clethodim, fluazifop-p-buthyl, fenoxaprop-p-ethyl, tepraloxydim, clethodim+fenoxaprop-p-ethyl e haloxyfop-methyl resultaram em controle eficiente em dois estádios de desenvolvimento (Adegas et al., 2010). Adegas et al. (2010) estudaram o controle de capim-amargoso na cultura da soja com o uso de herbicidas pós-emergentes alternativos ao glyphosate, e constataram que na fase inicial de desenvolvimento do capim-amargoso os herbicidas clethodim, fluazifop-p-buthyl, fenoxaprop-p-ethyl, tepraloxydim, clethodim+fenoxaprop-p-ethyl, paraquat, haloxyfop-methyl e imazapyr resultaram em controle eficaz, e em estádio mais avançado de desenvolvimento, o grupo de tratamentos mais eficaz foi formado por clethodim, fluazifop-p-buthyl, tepraloxydim, haloxyfop-methyl e paraquat.

Os herbicidas inibidores da ACCase, de forma geral, são adsorvidos a matéria orgânica e argilas do solo, já que possuem altos Kow e conseqüentemente Koc (Vidal e Merotto Junior, 2001). Ainda, todos os inibidores da ACCase sofrem biodegradação no solo, apresentando baixa persistência no ambiente (Vidal e Merotto Junior, 2001), o que torna seu efeito residual inexpressivo, necessitando de herbicidas pré-emergentes em mistura para manter um bom controle dos novos fluxos de germinação ao longo do período de crescimento e desenvolvimento da cultura.

Os resultados obtidos pelo experimento com herbicidas alternativos pré-emergentes mostraram que os herbicidas atrazine (2.500 $\mathrm{g} \mathrm{ha}^{-1}$ i.a.), Isoxaflutole (60 $\mathrm{g} \mathrm{ha}^{-1}$ i.a.), S-metolachlor (1.440 $\mathrm{g} \mathrm{ha}^{-1}$ i.a.), clomazone (1.000 $\mathrm{g} \mathrm{ha}^{-1}$ i.a.), diuron $\left(2.000 \mathrm{~g} \mathrm{ha}^{-1}\right.$ i.a. $)$ e flumioxazin $\left(50 \mathrm{~g} \mathrm{ha}^{-1}\right.$ i.a. $)$ foram eficientes para o controle de $D$. insularis até 80 dias após a aplicação dos mesmos. O herbicida amicarbazone (280 $\mathrm{g} \mathrm{ha}^{-1}$ i.a.) não apresentou controle satisfatório para o capim-amargoso até os 80 dias após a aplicação. Os resultados estão apresentados na Tabela 6.

Tabela 6. Controle percentual de capim-amargoso (Digitaria insularis) avaliado aos 20, 60 e 80 dias após a aplicação dos tratamentos herbicidas pré-emergentes (DAA), de forma visual e pesagem.

\begin{tabular}{|c|c|c|c|c|c|}
\hline \multirow{3}{*}{ Tratamentos } & \multirow{3}{*}{$\frac{\text { Dose }}{\left(\mathrm{g} \mathrm{ha}^{-1} \text { i.a. }\right)^{1}}$} & \multicolumn{4}{|c|}{ Capim-amargoso (Digitaria insularis) } \\
\hline & & \multicolumn{4}{|c|}{ Avaliações de controle (\%) } \\
\hline & & 20 DAA* & 60 DAA* & 80 DAA* & MS $^{2}$ \\
\hline 1- Testemunha sem aplicação & - & $0 \mathrm{c}$ & $0 \mathrm{c}$ & $0 \mathrm{c}$ & $13,46 \mathrm{c}$ \\
\hline 2 - Amicarbazone $\mathrm{M}^{\mathrm{M}}$ & 280 & $85 \mathrm{~b}$ & $42,5 \mathrm{~b}$ & $22,5 \mathrm{~b}$ & $4,20 \mathrm{~b}$ \\
\hline 3 - Atrazine ${ }^{\mathrm{M}}$ & 2500 & $100 \mathrm{a}$ & $100 \mathrm{a}$ & $97,5 \mathrm{a}$ & $0 \mathrm{a}$ \\
\hline 4 - Isoxaflutole ${ }^{\mathrm{MA}}$ & 60 & $100 \mathrm{a}$ & $83,7 \mathrm{a}$ & $88,7 \mathrm{a}$ & $0,41 \mathrm{a}$ \\
\hline $5-\mathrm{S}$-metolachlor ${ }^{\mathrm{M}}$ & 1440 & $100 \mathrm{a}$ & $100 \mathrm{a}$ & $100 \mathrm{a}$ & $0 \mathrm{a}$ \\
\hline $6-$ Clomazone $^{\mathrm{A}}$ & 1000 & $95 \mathrm{ab}$ & $97,5 \mathrm{a}$ & $98,7 \mathrm{a}$ & $0,01 \mathrm{a}$ \\
\hline $7-$ Diuron $^{\mathrm{A}}$ & 2000 & $100 \mathrm{a}$ & $100 \mathrm{a}$ & $100 \mathrm{a}$ & $0 \mathrm{a}$ \\
\hline 8 - Flumioxazin ${ }^{\mathrm{A}}$ & 50 & $91,3 \mathrm{ab}$ & $88,7 \mathrm{a}$ & $85 \mathrm{a}$ & $0,33 \mathrm{a}$ \\
\hline DMS $^{3}$ & & 14,96 & 18,46 & 19,35 & 3,46 \\
\hline CV $(\%)^{4}$ & & 7,52 & 10,16 & 11,01 & 63,42 \\
\hline
\end{tabular}

${ }^{1}$ gramas de ingrediente ativo por hectare; ${ }^{2}$ massa seca em gramas aos 80 DAA; ${ }^{3}$ Diferença mínima significativa; ${ }^{4}$ Coeficiente de variação; *Dias após a aplicação; ${ }^{\mathrm{M} H e r b i c i d a ~ r e c o m e n d a d o ~ p a r a ~ a ~ c u l t u r a ~ d o ~ m i l h o ; ~}{ }^{\mathrm{A}}$ Herbicida recomendado para a cultura do algodão; ${ }^{\mathrm{M}} \mathrm{Herbicida}$ recomendado para as culturas do milho e algodão. 
A integração entre herbicidas pré-emergentes e pós-emergentes pode resultar em melhor controle das plantas daninhas do que apenas uma aplicação em pós ou apenas uma aplicação em pré-emergência. Jones et al. (2001) chegaram à conclusão de que a aplicação da atrazine em milho resistente a glufosinate durante a semeadura da cultura, complementada pelo amônio-glufosinato para o controle das plantas escapes, resultou em melhor controle das plantas daninhas do que a aplicação de glufosinate em pós-emergência sem atrazine na semeadura. A aplicação do herbicida pré-emergente, complementado posteriormente com a aplicação de glyphosate em pós-emergência resulta em maior produtividade e menor presença de plantas daninhas, quando se compara com apenas a aplicação de glyphosate na pós-emergência (Ben et al., 2012; Toledo et al., 2015).

O uso de mais de um mecanismo de ação no controle de uma planta daninha problema é recomendado tanto para evitar o aparecimento de biótipos resistentes como para remediar a seleção já ocorrida, de modo que essa diversificação de modos de ação resulta na redução do banco de sementes do solo (Norsworthy et al., 2012). Tendo em vista o controle eficaz apresentado por alguns herbicidas avaliados neste experimento, o manejo da espécie $D$. insularis resistente ao glyphosate se torna possível em áreas de produção de milho ou algodão.

\section{Conclusões}

Foram determinadas alternativas de controle químico para o biótipo de Digitaria insularis resistente ao glyphosate, sendo elas, na pós-emergência da planta daninha no estádio de 1 a 2 perfilhos, os herbicidas nicosulfuron (600 $\mathrm{g} \mathrm{ha}^{-1}$ i.a.), imazapic + imazapyr (52,5 + 17,5 $\mathrm{g} \mathrm{ha}^{-1}$ i.a.), atrazine (3000 $\mathrm{g} \mathrm{ha}^{-1}$ i.a.), haloxifop-methyl (60 $\mathrm{g} \mathrm{ha}^{-1}$ i.a.) e tepraloxydim (100 $\mathrm{g} \mathrm{ha}^{-1}$ i.a.). Os herbicidas atrazine ( $2500 \mathrm{~g} \mathrm{ha}^{-1}$ i.a.), isoxaflutole (60 $\mathrm{g} \mathrm{ha}^{-1}$ i.a.), S-metolachlor (1440 $\mathrm{g} \mathrm{ha}^{-1}$ i.a.), clomazone (1000 $\mathrm{g} \mathrm{ha}^{-1}$ i.a.), diuron (2000 $\mathrm{g} \mathrm{ha}^{-1}$ i.a.) e flumioxazin (50 $\mathrm{g} \mathrm{ha}^{-1}$ i.a.) se apresentaram como pré-emergentes eficazes para o controle desta espécie.

\section{Referências}

Adegas, F.S.; Gazziero, D.L.P.; Voll, E.; Osipe, R. Alternativas de controle químico de Digitaria insularis resistente ao herbicida glyphosate. In: Congresso Brasileiro da Ciência das Plantas Daninhas, 27., 2010, Ribeirão Preto. Anais... Ribeirão Preto: SBCPD, 2010. n.161.

Andrei, E. Compêndio de defensivos agrícolas: guia prático de produtos fitossanitários para uso agrícola. 8.ed. São Paulo: Andrei, 2009. 1380p.

Barroso, A.A.M.; Albrecht, A.J.P.; Reis, F.C.; Victoria Filho, R. Interação entre herbicidas inibidores da ACCase e diferentes formulações de glyphosate no controle de capim-amargoso. Planta Daninha, v.32, n.3, p.619-627, 2014.

Ben, R.; Inoue, M.; Cavalcante, N.; Mendes, K.; Dallacort, R.; Santos, E. Eficácia do glufosinato de amônio associado com outros herbicidas na cultura do algodão Liberty Link ${ }^{\circledR}$. Revista Brasileira de Herbicidas, v.11, n.3, p.249-257, 2012.

Duke, S.O.; Powles, S.B. Glyphosate: a once-ina-century herbicide. Pest Management Science, v.64, p.319-325, 2008.

Ferreira, C.; Osipe, J.B.; Alves, K.A.; Sorace, M.A.; Osipe, R.; Brito Neto, A.J. Avaliação da eficiência do herbicida Finale (amônio glufosinato) aplicado na modalidade seqüencial, no controle químico de buva, na operação de manejo em plantio direto, da cultura da soja. In: Congresso Brasileiro da Ciência das Plantas Daninhas, 27., 2010, Ribeirão Preto. Anais.... Ribeirão Preto: SBCPD, 2010. n.233.

Frans, R.; Talbert, R.; Marx, D.; Crowley, H. Experimental design and techniques for measuring and analyzing plant responses to weed control practices. In: Camper, N.D. (Ed.). Research methods in weed science. 3.ed. Champaign: Southern Weed Science Society, 1986. p.29-46. 
Frisvold, G.B.; Hurley, T.M.; Mitchel, P.D. Adoption of best management practices to control weed resistance by corn, cotton, and soybean growers. AgBioForum, v.12, p.370-381, 2009.

Gemelli, A.; Oliveira Junior, R.S.; Constantin, J.; Braz, G.B.P.; Jumes, T.M.C.; Gheno, E.A.; et al. Estratégias para o controle de capim-amargoso (Digitaria insularis) resistente ao glyphosate na cultura milho safrinha. Revista Brasileira de Herbicidas, v.12, n.2, p.162-170, 2013.

Gemelli, A.; Oliveira Junior, R.S.; Constantin, J.; Braz, G.B.P.; Jumes, T.M.C.; Oliveira Neto, A.M.; et al. Aspectos da biologia de Digitaria insularis resistente ao glyphosate e implicações para o seu controle. Revista Brasileira de Herbicidas, v.11, n.2, p.231-240, 2012.

Heap, I. The international survey of herbicide resistant weeds. Disponível em: $<$ www.weedscience. com>. Acesso em: 07 mar. 2017.

Hurtley, T.M.; Mitchell, P.D.; Frisvold, G.B. Weed management costs, weed best management practices, and the Roundup Ready weed management program. AgBioForum, v.12, p.281-290, 2009.

Jones, C.A.; Chandler, J.M.; Morrison Junior, J.E.; Senseman, S.A.; Tingle, C.H. Glufosinate combinations and row spacing for weed control in glufosinate-resistant corn (Zea mays). Weed Technology, v.15, p.141-147, 2001.

Lingenfelter, D.D.; Curran, W.S. Effect of Glyphosate and Several Accase-inhibitor Herbicides on Wirestem Muhly (Muhlenbergia Frondosa) Control. Weed Technology, v.21, n.3, p.732-738, 2007.

Llewellyn, R.S. Information quality and effectiveness for more rapid adoption decisions by producers. Field Crops Research, v.104, p.148-156, 2007. MAPA - Ministério da agricultura Pecuária e Abastecimento. SISLEGIS: Sistema de Consulta à Legislação. Disponível em: $<$ http://sistemasweb. agricultura.gov.br/sislegis/ action/detalhaAto. do? method $=$ consultarLegislacaoFederal $>$. Acesso em: 07 jun. 2017.

Melo, M.S.C. Levantamento de ocorrência, alternativas de manejo, mecanismos de resistência e herança genética do capim-amargoso (Digitaria insularis) resistente ao herbicida glyphosate. 2015. 108 f. Tese (Doutorado em Fitotecnia) Universidade de São Paulo, Escola Superior de Agricultura “Luiz de Queiroz”, Piracicaba, 2015.

Melo, M.S.C.; Rosa, L.E.; Brunharo, C.A.C.G.; Nicolai, M.; Christoffoleti, P.J. Alternativas para o controle químico de capim-amargoso (Digitaria insularis) resistente ao glyphosate. Revista Brasileira de Herbicidas, v.11, n.2, p.195-203, 2012.

Moreira, M.S.; Carvalho, S.J.P.; Nicolai, M.; Christoffoleti, P.J. Resistência de Conyza canadensis e Conyza bonariensis ao herbicida glyphosate. Planta Daninha, v.25, n.1, p.157-164, 2007.

Norsworthy, J.K.; et al. Reducing the risks of herbicide resistance: best management practices and recommendations. Weed Science, v.60, p.31-62, 2012.

Parreira, M.C.; Espanhol, M.; Duarte, D.J.; Correia, N.M. Manejo químico de Digitaria insularis em área de plantio direto. Agrária, v.5, n.1, p.13-17, 2010.

Powles, S.B. Evolved glyphosate-resistant weeds around the world: lessons to be learned. Pest Management Science, v.64, p.360-365, 2008.

Powles, S.B.; Yu, Q. Evolution in Action: Plants Resistant to Herbicides. Annual Review of Plant Biology, v.61, p.317-347, 2010.

SBCPD - Sociedade Brasileira da Ciência das Plantas Daninhas. Procedimentos para instalação, avaliação e análise de experimentos com herbicidas. Londrina: SBCPD, 1995. 42p.

Timossi, P.C. Manejo de rebrotes de Digitaria insularis (L.) Fedde no plantio direto de milho. Planta Daninha, v.27, n.1, p.175-179, 2009. 
Toledo, R.; Silva Junior, A.; Negrisoli, R.; Negrisoli, E.; Corrêa, M.; Rocha, M.; Victória Filho, R. Herbicidas aplicados em pré-emergência para o controle de Ipomoea spp. na cultura de canade-açúcar em época seca. Revista Brasileira de Herbicidas, v.14, n.4, p.271-279, 2015.

Vargas, L.; Roman, E.S.; Rizzardi, M.A.; Silva, V.C. Alteração das características biológicas dos biótipos de azevém (Lolium multiflorum) ocasionada pela resistência ao herbicida glyphosate. Planta Daninha, v.23, n.1, p.153-160, 2005.

Vargas, L.; Roman, E.S.; Rizzardi, M.A.; Toledo, R.E.B. Manejo de azevém resistente ao glyphosate em pomares de maçã com herbicida select (clethodim). Revista Brasileira de Herbicidas, n.1, p.30-36, 2006.
Vidal, R.; Merotto Junior, A. Herbicidas inibidores da ACCase. In: Vidal, R.; Merotto Junior, A. (Ed.). Herbicidologia. Porto Alegre: UFRS, 2001. p.15-24.

Webster, T.M.; Sonoskie, L.M. Loss of Glyphosate Efficacy: a Changing Weed Spectrum in Georgia Cotton. Weed Science, v.58, p.73-79, 2010.

Wilson, R.S.; Tucker, M.A.; Hooker, N.H.; Lejeune, J.T.; Doohan, D. Perceptions and beliefs about weed management: perspectives of Ohio grain and produce farmers. Weed Technology, v.22, p.339-350, 2008.

Yamauti, M.S.; Barroso, A.A.M.; Souza, M.C.; Alves, P.L.C.A. Controle químico de biótipos de buva (Conyza canadensis e Conyza bonariensis) resistentes ao glyphosate. Revista Ciência Agronômica, v.41, n.3, p.495-500, 2010. 